\title{
IAMJ
}

INTERNATIONAL

AYURVEDIC

MEDICAL JOURNAL

\section{COMPREHENSIVE STUDY OF BALYA MAHAKASHAYA ON THE BASIS OF THEIR RASAPANCHAK IN RELATION TO NIGHANTU: A REVIEW}

\author{
$\underline{\text { Bhagat Seema }}^{1}, \underline{\text { Ramamurthy Aku}}^{2}$, $\underline{\text { Rathore Poonam }}^{3}$ \\ ${ }^{1} \mathrm{MD}$ Scholar, ${ }^{2}$ Professor, ${ }^{3} \mathrm{MD}$ Scholar \\ Postgraduate Department of Dravyaguna \\ National Institute of Ayurveda, Madav Vilas Palace, Amer Road-Jaipur (302002), Rajasthan, India
}

Corresponding Author: bhagatseema033@gmail.com

\section{https://doi.org/10.46607/iamj2809072021}

(Published Online: July 2021)

Open Access

(C) International Ayurvedic Medical Journal, India 2021

Article Received: 28/06//2021 - Peer Reviewed: 02/07/2021 - Accepted for Publication: 04/07/2021

\section{Check for updates}

\begin{abstract}
An impressive number of thousands of plants have been utilising for the treatment of diseases for thousands of years. Many of them are clearly explained in Ayurveda. In Ayurveda certain drugs manifest their action by their Rasa (taste); some by their Virya (potency) or other qualities, some by Vipaka (biological transformation) and others by their specific action. As per their Raspanchak, some of these drugs possesses an affinity for bala. The drugs that provide Bala (strength) and vitality to the body have been grouped under Balya mahakashaya by Acharya Charak. Bala (strength) plays an important role in the diagnosis and treatment of various diseases. This study aimed to discuss the details of ten Balya Mahakashya drugs based on their Raspanchak i.e Rasa, Guna, Virya, Vipaka and Karma and in relation to Bhavaprakash Nighantu, Dhanwantari Nighantu, Raj Nighantu and Kaiyadev Nighantu.
\end{abstract}

Keywords: Bala, Balya Mahakashaya, Raspanchak, Nighantu. 


\section{INTRODUCTION}

The action Balya is defined as "Balaaya Hitam Balyam" which refers to substances that are beneficial and tend to increase Bala of an individual. The concept of Bala has been well explained by different Acharaya in Ayurveda. Acharya Charak has classified the drugs according to their Karma (pharmacological properties) into 50 groups or Mahakashaya. Each Mahakashaya contains 10 drugs possessing similar properties. Balya Mahakashaya is one of them containing 10 drugs; Aindri, Rishabhi, Atirasa, Rishyaprokta, Payasya, Ashvagandha, Sthira, Rohini, Bala, Atibala. All these drugs tend to increase the vitality and strength of the body. In Ayurveda, the Bala is used in various contexts. According to Acharya Charak: There are 3 types of Bala (Strength) namely, Sahej, Kalaja and Yuktikruta ${ }^{1}$ Sahej Bala (congenital) is that which is natural to the body and mind. It exists in the mind and body since birth. Kalaja Bala (time-effected) depends on Ritu (seasonal variation) and Vaya (age-factor). Yuktikruta (Acquired) Bala (strength) can be produced by proper application of diet and exercise.

Acharya Charak has also explained the 13 factors that are responsible for the promotion of Bala (Strength) which are explained below as ${ }^{2}$

1. Birth in a country where people are naturally strong.

2. Birth at a time when people naturally gain strength.

3. Favourable disposition of time (pleasant and moderate climate).

4. The excellence of the qualities of the seed (sperm) and Aasaya (ovum and uterus) of the parents.

5. The excellence of the ingested food.

6. The excellence of the physique.

7. The excellence of the Satmya (wholesomeness of various factors responsible for the maintenance of the body)

8. The excellence of the mind.

9. Favourable disposition of nature.

10. Exercise

11. Cheerful disposition.
It implies that inhabitants of certain places like Sindh are strong by nature. This is because of the specific characteristics of those places. Birth in such countries makes the man strong. Similarly, in certain seasons like Hemanta (November to January) and Sisira (January to March), people generally gain strength, and birth in such seasons makes the individual strong. Moderate climate, when there is no excess of heat and cold and which is pleasant, also promotes the strength of the individual. The progeny of parents having no abnormality or having the excellence of sperm, ovum and uterus is endowed with strength. The mind also controls the body. If the individual is having a soul mind, then his body will also be quite strong. Some people are also endowed with a strong physique because of their virtuous actions in a past life. Habitual performance of exercise is also known to promote the strength of the individual. In Sushruta Samhita, the word Bala refers to Ojas which has the function of maintaining the Bala of the body. ( $\mathrm{Su}$. Su.15/24). Acharaya Sushruta has also mentioned that the strength bestows stability and growth of muscles, ability to perform all activities without any hindrance, clarity of voice, the brightness of colour/complexion and ability of external sense organs (means Karmaendriyas or motor organs i.e hands, feet, pharynx, penis, and anus) and internal sense organs (Janaendriyas or sensory organs i.e. eyes, ears, tongue, nose and skin) and also Manas (mind) to perform their functions ${ }^{3}$. In this study, Raspanchak of Balya Mahakasahya drugs in different Nighantus are discussed to elucidate the Balya Karma of these drugs.

AIM AND OBJECTIVE: To compile and elucidate the Balya Karma of Balya Mahakashya drugs based on their Raspanchak in relation to Nighantu.

MATERIALS AND METHODS: Detailed study of Ayurvedic texts i.e Charaka Samhita, Sushruta Samhita, Nighantu, information from various National and International Journals, peer-reviewed papers, Pub Med, were used for the better understanding of the Balya Mahakashya drugs. 
Table 1: Description of Balya Mahakashaya drugs: Botanical name, Family and Useful part

\begin{tabular}{|l|l|l|l|l|}
\hline S.No. & Drugs & Botanical Name & Family & Useful Part \\
\hline 1. & Aindri & Herpestis monnier Linn. & Scrophulariaceae & Whole plant \\
\hline 2. & Rishabhi & Mucuna pruriens Bek. & Fabaceae & Seed \\
\hline 3. & Atirasa & Asparagus racemosus Willd. & Liliaceae & Root \\
\hline 4. & Rishyaprokta & Teramnus labialis Spreng. & Fabaceae & Root \\
\hline 5. & Payasya & Ipomea digitata Linn. & Convolvulaceae & Root \\
\hline 6. & Asvagandha & Withania somnifera Dunal. & Solanaceae & Root \\
\hline 7. & Sthira & Desmodium gangeticum DC. & Papilionaceae & Root \\
\hline 8. & Rohini & Picrorhiza kurroa Royale ex Benth. & Scrophulariaceae & Rhizome \\
\hline 9. & Bala & Sida cordifolia Linn. & Malvaceae & Root \\
\hline 10. & Atibala & Abutilon indicum Linn. & Malvaceae & Root \\
\hline
\end{tabular}

Table 2: Properties of Balya Mahakashaya as per Bhavprakash Nighantu

\begin{tabular}{|c|c|c|c|c|c|c|c|}
\hline S.No. & Drugs & Varga & Rasa & Guna & Virya & Vipaka & Karma \\
\hline 1. & Aindri & Guduchyadi & $\begin{array}{l}\text { Tikta, } \\
\text { Kashaya, } \\
\text { Madhura }\end{array}$ & Laghu & Shita & Madhura & Ayushya, Rasayana, Medhakar \\
\hline 2. & Rishabhi & Guduchyadi & $\begin{array}{l}\text { Madhura, Tik- } \\
\text { ta }\end{array}$ & Guru & - & - & $\begin{array}{l}\text { Vrishya, Vatnashaka, Balya, } \\
\text { Raktadoshanashak }\end{array}$ \\
\hline 3. & Atirasa & Guduchyadi & $\begin{array}{l}\text { Tikta, } \\
\text { Madhura }\end{array}$ & Guru & Shita & - & $\begin{array}{l}\text { Rasayana, Medhakara, Balya, } \\
\text { Tridoshahara }\end{array}$ \\
\hline 4. & Rishyaprokta & Guduchyadi & $\begin{array}{l}\text { Tikta, } \\
\text { Madhura }\end{array}$ & Ruksha & Shita & - & $\begin{array}{l}\text { Grahi, } \\
\text { Kaphakaraka }\end{array}$ \\
\hline 5. & Payasya & Guduchyadi & Madhura & $\begin{array}{l}\text { Snigdha, } \\
\text { Guru }\end{array}$ & Shita & - & Satnyashukarakara, Rasayana \\
\hline 6. & Asvagandha & Guduchyadi & Tikta, Kashya & & Ushna & - & $\begin{array}{l}\text { Shukravardhaka, } \quad \text { Vata- } \\
\text { Kaphadoshaagana }\end{array}$ \\
\hline 7. & Sthira & Guduchyadi & $\begin{array}{l}\text { Tikta, } \\
\text { Madhura }\end{array}$ & Guru & - & - & Tridoshanashaka, Kriminashaka \\
\hline 8. & Rohini & Haritakiyadi & Tikta & $\begin{array}{l}\text { Ruksha, } \\
\text { laghu }\end{array}$ & - & Katu & $\begin{array}{l}\text { Bhedan, Deepan, Hridya, } \\
\text { Kapha-piitajwara, Kriminasha- } \\
k a\end{array}$ \\
\hline 9. & Bala & Guduchyadi & Madhura & Snighdha & Shita & Madhura & Balya, Grahi, Vatahara \\
\hline 10. & Atibala & Guduchyadi & Madhura & Snighdha & Shita & Madhura & Balya, Grahi,Vatahara \\
\hline
\end{tabular}

Table 3: Properties of Balya Mahakashya as per Dhanwantari Nighantu

\begin{tabular}{|l|l|l|l|l|l|l|l|}
\hline S.No. & Drugs & Varga & Rasa & Guna & Virya & Vipaka & Karma \\
\hline 1. & Aindri & - & \multicolumn{1}{|l}{-} & - & & - & - \\
\hline 2. & Rishabhi & Guduchyadi & $\begin{array}{l}\text { Madhura, Tik- } \\
\text { ta }\end{array}$ & - & Shita & - & Vatahara, Vrishya \\
\hline 3. & Atirasa & Guduchyadi & $\begin{array}{l}\text { Tikta, } \\
\text { Madhura }\end{array}$ & - & Shita & - & Vata-pittahara, Vrishya, Rasayana \\
\hline 4. & Rishyaprokta & Guduchyadi & $\begin{array}{l}\text { Madhura, Tik- } \\
\text { ta }\end{array}$ & Shita & - & Daha, Jwara, Anilhara \\
\hline 5. & Payasya & Guduchyadi & Madhura & - & & - & Balya, Vata-pittahara, Dhatu- \\
\hline
\end{tabular}




\begin{tabular}{|l|l|l|l|l|l|l|l|}
\hline & & & & & vardhak \\
\hline 6. & Asvagandha & Guduchyadi & Kashaya, Tikta & - & Ushna & - & Kapha-vatahara, Balya \\
\hline 7. & Sthira & Guduchyadi & Tikta & Guru & Ushna & - & Vishmajwaranashaka \\
\hline 8. & Rohini & Guduchyadi & Tikta, Katu & - & Shita & - & Vishmajwara, Aruchi nashaka \\
\hline 9. & Bala & Guduchyadi & Madhura & Snigdha & Shita & Madhura & Balya, Ojavardhan \\
\hline 10. & Atibala & Guduchyadi & Madhura & Snigdha & Shita & Madhura & Vrishya, Balya \\
\hline
\end{tabular}

Table 4: Properties of Balya Mahakashya as per Raj Nighantu ${ }^{6}$

\begin{tabular}{|c|c|c|c|c|c|c|c|}
\hline S.No. & Drugs & Varga & Rasa & Guna & Virya & Vipaka & Karma \\
\hline 1. & Aindri & - & - & - & - & - & - \\
\hline 2. & Rishabhi & Guduchyadi & Madhura & - & - & - & Vrishya, Vataroganashak \\
\hline 3. & Atirasa & Shatahwadi & Madhura & Laghu & Shita & - & $\begin{array}{l}\text { Rasayana, } \quad \text { Kapha-pittahara, } \\
\text { Vrishya }\end{array}$ \\
\hline 4. & Rishyaprokta & Guduchyadi & Tikta & - & - & - & Balya, vrishya, pushtikara \\
\hline 5. & Payasya & Moolakadi & $\begin{array}{l}\text { Madhura, } \\
\text { Amal, kashaya, } \\
\text { Tikta }\end{array}$ & - & - & - & Pittadoshahara, Shoohara \\
\hline 6. & Asvagandha & Shatahwadi & Katu, kashaya & - & Ushna & - & Balya, Vatahara \\
\hline 7. & Sthira & Shatahwadi & Tikta & Guru & Ushna & - & Vatadoshahara \\
\hline 8. & Rohini & Pippalyadi & Katu, Tikta & - & - & - & Shitapittahara \\
\hline 9. & Bala & Shatahwadi & Tikta, Madhura & - & - & Madhura & $\begin{array}{l}\text { Balya, Viryaprada, Kapharoga } \\
\text { nashak }\end{array}$ \\
\hline 10. & Atibala & Shatahwadi & Tikta, Katu & - & Shita & - & Vatanashak, Kledanashak \\
\hline
\end{tabular}

Table 5: Properties of Balya Mahakashaya as per Kaiydeva Nighantu ${ }^{7}$

\begin{tabular}{|l|l|l|l|l|l|l|l|}
\hline S.No. & Drugs & Varga & Rasa & Guna & Virya & Vipaka & Karma \\
\hline 1. & Aindri & - & - & - & - & - & - \\
\hline 2. & Rishabhi & Aushadhi & Madhura, Tikta & Guru & Shita & - & Brighana, Vrishya, tridoshahara \\
\hline 3. & Atirasa & Aushadhi & Tikta, Madhura & Snigdha & Shita & - & Balya, medhya \\
\hline 4. & Rishyaprokta & Aushadhi & Madhura & Ruksha & Shita & Madhura & Vata-pittahara, Shukrala \\
\hline 5. & Payasya & Aushadhi & Madhura & Guru & Shita & Madhura & Vrishya, Rasayana \\
\hline 6. & Asvagandha & Aushadhi & Kashaya, Tikta & - & Ushna & - & $\begin{array}{l}\text { Vrishya, Balya, kaphahara, Ra- } \\
\text { sayana }\end{array}$ \\
\hline 7. & Sthira & Aushadhi & Madhura, Tikta & Guru & Ushna & - & Rasayana, Vrishya, tridoshahara \\
\hline 8. & Rohini & Aushadhi & Katu, Tikta & $\begin{array}{l}\text { Laghu, } \\
\text { ruksha }\end{array}$ & Shita & Katu & $\begin{array}{l}\text { Bhedana, } \\
\text { pittahara }\end{array}$ \\
\hline 9. & Bala & Aushadhi & Madhura & Snigdha & Shita & Madhura & Oja-balvardhana \\
\hline 10. & Atibala & Aushadhi & Madhura & Snigdha & Shita & Madhura & Ayushya, Kantivardhana \\
\hline
\end{tabular}

\section{DISCUSSION}

Bala (strength) deteriorates day by day in today's fast pace of life where every individual is facing anxiety, stress, insomnia, depression, mental illness etc. all these factors attenuate the individual's both physical and mental strength. For improving the strength of many nutritional supplements, tonics are mentioned in modern medicine. In Ayurveda, Balya Mahakashaya has mentioned for improving Bala. The delineation of Balya Mahakashaya in different Nighantus showed that they are having mainly Madhura Rasa (sweet) then Tikta Rasa (bitter), Kashaya Rasa (astringent), Katu Rasa (pungent), as secondary Rasa, Guru, Snigdha Guna mainly, Shita Virya (potency), 
Madhura Vipaka (biotransformation of drug inside the body) and Karma (pharmacological properties) like Balya (increases vitality or strength of the body), Vrishya (aphrodisiac), Ayushykara (increases life span), Oja Vardhan (increases the immunity against diseases) Rasayana, Deepana (intensifies digestive fire) Vatahara (which attenuates the excess Vata dosha) , Pushtikara (nourishes the body). Madhura Rasa helps in the Poshana of all Dhatus viz. Rasa, Rakta, Mamas, Meda, Asthi, Majja, Shukra, Ayushya, Balkaraka (increases strength), Tarpan (nourishes the body), Brhmana (give bulkiness), prinan (nourishes all the parts of the body) (Ch. Su.26/43), Shodhana of all channels (Srotas) by Tikta Rasa and its Ushna guna, Deepan (appetizer), Pachana (digestive) Rochana, Sodhana (eliminates unwanted material from body) Guna of both Tikta and Katu Rasa. (S.Su.42/9) and its Medhya (promotes intelligence) property of tikta rasa (As.Hr.Su.10/14-16). All these properties attribute greatly towards the Balya Karma of Balya Mahakashaya drugs due to their Rasa, Guna Virya, Vipaka. Many research works have been done on the drugs of Balya Mahakashaya as Herpestis monniera or Bacopa monnieri Linn. has been reported as a neural, cardiac tonic, memory enhancer, anti-depressant, anti-anxiety activity, anti-epileptic activity, anticancer activity ${ }^{8}$. It is reported that Mucuna pruriens Bek. possesses neuroprotective activity, antimicrobial activity, antioxidant activity, anti-diabetic activity ${ }^{9}$. Methanolic extract of Asparagus racemosus Willd. has significant activities like antitussive activity, and also possesses adaptogenic, activity, aphrodiasiac activity, protect against amnesia enhances memory activity, anti-stress activity, neurodegenerative activity, cardio protective activity ${ }^{10}$. The experimental study of Teramnus labialis Spreng with aqueous extract $500 \mathrm{mg} / \mathrm{kg}$ body weight and ethanol extract $500 \mathrm{mg} / \mathrm{kg}$ body weight shows that the aqueous extract of Mashparni (Teramnus Labialis Spreng) shows a significant immune-modulatory action compared to its ethyl extract ${ }^{11}$. Ipomea digitata Linn. has been reported to possess a wide range of pharmacological activities like anti-diabetic, anti-oxidant potential, Galactagogue activity (breastfeeding/ Lacta- tion), hepatoprotective activity, hypertension, blood pressure and another cardiovascular risk, indigestion, infertility, Revitalizing effect, Spasmogenic effect $^{12}$.Phytochemical studies on Withania Somnifera (L.) Dunal revealed the presence of important chemical constituents such as flavonoids, phenolic acids, alkaloids, saponins, tannins, and with anolides shows various pharmacological activities like anti-cancer, immunomodulatory, cardioprotective, neuroprotective, anti-ageing, anti-stress/adaptogenic and antidiabetic. Various clinical trials show that the plant extract and its bioactive compounds are used in the prevention and treatment of many diseases like amnesia, anxiety, cancer, neurogenerative ${ }^{13}$. Desmodium Gangeticum DC. has pharmacological activities like analgesic activity, anti-inflammatory activity, antinociceptive activity, anti- amnesic activity, antioxidant activity, anti-pyretic activity ${ }^{14}$. Picrorhiza kurroa Royale ex Benth possesses antioxidant activity, immunomodulatory activity, anti-cholestatic activity, anti-inflammatory activity and its glycoside kutkin possess significant hepatoprotective action ${ }^{15}$. Leaves of Sida cordifolia Linn. contain small quantities of ephedrine and pseudoephedrine which are helpful in asthma, fat loss, to increase energy ${ }^{16}$. And also possess pharmacological activities like CNS depressant, adaptogenic activity, anti-microbial activity, antioxidant activity, Anti Parkinson's disease ${ }^{17}$.Abutilon Indicum possesses the antioxidant potential and immunomodulatory activity ${ }^{18}$.

\section{CONCLUSION}

The above-mentioned discussion clearly explains the importance of Balya Mahakahaya drugs possessing the ability to increase the physical, mental strength, vitality of the body and immunity against diseases. This study will help to strengthen the existing knowledge of Balya Mahakashaya drugs and helps to create novel therapeutic uses that can be used for better health care in the future.

\section{REFERENCES}


1. Sharma Priyavrat, Charak Samhita Text with English translation, Vol-1, Varanasi Chaukhambha Orientalia, 2014, Pageno.75

2. Sharma Ram Karan and Dash Bhagwan, Agnivesa Caraka Samhita Text with English Translation \& Critical Exposition based on Cakrapani Datta's Ayurveda Dipika, Vol-2, Varanasi; Chowkhamba Sanskrit Series Office; Reprint year: 2017.page no. 436-437

3. Murthy K.R. Srikantha, Susruta Samhita, Text English Translation, Notes, Appendices and Index, Vol-1, Edition first, Varanasi; Chaukhambha Orientalia; 2000. page no.104

4. Chunekar, K.C Bhavaprakash Nighantu (Indian Material Medica), Varanasi; Chaukambha Bharti Academy; Reprint 2018. p.no 67-447

5. Priyavrat Sharma, Dhanwantari Nighantu, I $^{\text {st }}$ edition Varanasi; Chhukhambha Orientali; 1982.page no.2368

6. Tripathi Indradeo, Raj Nighantu of Pandit Narhari edited with Dravyagunaprakasita Hindi Commentary, Varanasi; krishandas Academy; page no.34-207.

7. Sharma Priyavrat, Sharma Guru Prasad Sharma Kaiyedeva Nighantu, Varanasi; Chukhambha Orientalia; 2013. page no.11-638

8. Shalini Lal and Binit Baraik, International Journal of Pharmaceutical Sciences and research, Phytochemical and pharmacological profile of Bacopa monnieri-An ethnomedicinal plant. 2019;10(3):1001-1013

9. Mukesh kumar Yadav, Prabhat Upadhyay, Suresh Purohit, B.L Pandey, Harish Shah. International journal of green pharmacy. Phytochemistry and pharmacological activity of Mucuna pruriens: A review.2017,11(2):69-72

10. Noorul Hasan, Nesar Ahmad, Shaikh Zohrameena, Mohd Khalid, Juber Akhtar. international journal of advanced research. Asparagus racemosus: for medicinal uses and pharmacological actions.2016,4(3):259267

11. Dr Shweta, Dr Seema Pradeep, Dr Shiva Manjunath M.P. Journal of Ayurveda and Integrated Medical Sciences. Immunomodulatory activity of MashparniTeramnus Labialis Spreng.2019, 4(6):13-18

12. Neha Rauniyar and Deepa Srivastava. The Journal of Indian Botanical Society. Ipomea Digitata: A Therapeutic Boon from Nature to Mankind.2020, 100(3\&4):185-191.

13. Pulok K. Mukherjee, Subhadip Banerjee, Sayan Biswas, Bhaskar Das, Amit Kar, C.K. Katiyar. With- ania Somnifera (L.) Dunal- Modern perspectives of an ancient Rasayana from Ayurveda.Journal of Ethnopharmacology.2021, Volume 264

14. Bhavesh Vaghela, Sandip Buddhadev, Leena Shukla. Pharmacological activities of Desmodium gangeticum: An Overview. An International Journal of Pharmaceutical Sciences.2013,4(4):264-277

15. K. Kant, M.Walia, V.K.Agnihotri, Vijaylata Pathania and B.Singh.Evaluation of Antioxidant activity of Picrorhiza Kurroa(leaves) extract.Indian Journal of Pharmaceutical Sciences. 2013,75(3):324-329

16. Ashwini Kumar Sharma. Medicinal properties of Bala (Sida Cordifolia Linn. and its species)

17. Ankit Jain, Shreya Choubey, P. K Singour, R.S. Pawar, H. Rajak.Journal of Applied Pharmaceutical Science.Sida Cordifolia (Linn.)-An Overview.2011,1(02):23-31

18. Sapna Choudhary, Satya Prakash Chaudhary, Sadhana Singh, Vinod Kumar Joshi. International Ayurvedic Medical Journal .2017 5(1); 282-287

\section{Source of Support: Nil Conflict of Interest: None Declared}

How to cite this URL: Bhagat Seema et al: Comprehensive Study Of Balya Mahakashaya On The Basis Of Their Rasapanchak In Relation To Nighantu: A Review. International Ayurvedic Medical Journal \{online\} 2021 \{cited July 2021\} Available from: http://www.iamj.in/posts/images/upload/1501_1506.pdf 